\title{
Electronic Structure and Spectra of $\mathrm{CuO}$
}

\author{
C. E. Ekuma, ${ }^{1,2, \text { f V I. Anisimov, }}{ }^{3,4, \text { J. Moreno, }},{ }^{1,2, \text { 用 and M. Jarrell }}{ }^{1,2,8}$ \\ ${ }^{1}$ Department of Physics \& Astronomy, Louisiana State University, Baton Rouge, LA 70803, USA \\ ${ }^{2}$ Center for Computation and Technology, Louisiana State University, Baton Rouge, LA 70803, USA \\ ${ }^{3}$ Institute of Metal Physics, Russian Academy of Sciences, 620990 Yekaterinburg, Russia \\ ${ }^{4}$ Ural Federal University, 620990 Yekaterinburg, Russia
}

\begin{abstract}
We report the electronic structure of monoclinic $\mathrm{CuO}$ as obtained from first principles calculations utilizing density functional theory plus effective Coulomb interaction (DFT $+\mathrm{U}$ ) method. In contrast to standard DFT calculations taking into account electronic correlations in DFT $+\mathrm{U}$ gave antiferromagnetic insulator with energy gap and magnetic moment values in good agreement with experimental data. The electronic states around the Fermi level are formed by partially filled $\mathrm{Cu}$ $3 d_{x^{2}-y^{2}}$ orbitals with significant admixture of $\mathrm{O} 2 p$ states. Theoretical spectra are calculated using DFT + U electronic structure method and their comparison with experimental photoemission and optical spectra show very good agreement.
\end{abstract}

PACS numbers: 71.27.+a,71.15.Mb,74.25.Jb,78.20.-e

\section{INTRODUCTION}

The cupric oxide $(\mathrm{CuO})$ system has been studied for decades both for fundamental understanding and applied reasons. It is generally recognized as the prototype material of a broad family of the strongly correlated (SC) oxides. 11 Although the local environment of $\mathrm{Cu}$ in the $\mathrm{Cu}-\mathrm{O}$ planes are strongly distorted, the planes share many commonalities to those thought to be responsible for superconductivity in the layered cuprate systems. 2, 3] A consequence of this, a good understanding of the electronic structure of $\mathrm{CuO}$ will play a key role in understanding and developing models for the normal state behavior of the high temperature superconducting cuprates. High-temperature superconductivity was discovered in the copper oxide perovskites as early as 1986, [4. 5] but its origin and mechanism are still under intensive debate.

$\mathrm{CuO}$ is an exceptional member of the generally, rocksalt family as it deviates both structurally and electronically from others as one traverses the periodic table from $\mathrm{MnO}$ to $\mathrm{CuO}$. Unlike other members of the $3 d$ transition oxides (TMO) which crystallizes in the cubic rocksalt structure (with possible rhombohedral distortions), 6 6 - 8 Tenorite $(\mathrm{CuO})$ crystallizes in the lower symmetry monoclinic $\left(\mathrm{C}_{2 h}^{6}\right)$ crystal strucutre. Also, Unlike other antiferromagnetic (AFM) semiconductors, with known disordered paramagnetic character above the Néel temperature, $\mathrm{CuO}$ behaves as a $1 \mathrm{D}$ antiferromagnet [9] with strong antiferromagnetic ordering especially along the (101̃) direction prevailing even above the Néel temperature of $231 \mathrm{~K}$. A 3D collinear antiferromagnetic order has been reported below $213 \mathrm{~K}$, [10] while between 213

\footnotetext{
*Electronic Address: cekuma1@lsu.edu

${ }^{\dagger}$ Electronic Address: via@imp.uran.ru

${ }^{\ddagger}$ Electronic Address: moreno@lsu.edu

$\S$ Electronic Address: jarrellphysics@gmail.com
}

$\mathrm{K}<\mathrm{T}_{N} \lesssim 231 \mathrm{~K}$, it is reported to have a $3 \mathrm{D}$ noncollinear antiferromagnetic order. [11] Also, $\mathrm{CuO}$ has substantially lower than expected Néel temperatue $\mathrm{T}_{N}$ $\approx 230 \mathrm{~K}$, following a simple linear extrapolation of the trend of other TMOs across the periodic table. However, like other TMOs, $\mathrm{CuO}$ is an antiferromagnetic insulator. [6, 8, 12, 13

Aside the fundamental importance of $\mathrm{CuO}$ in understanding the properties of high-temperature superconductivity, it has other important practical technological applications. $\mathrm{CuO}$ has found practical applications in areas such as gas sensor, 14] solar cells and photovoltaics, [15, 16] catalysts, [17 varistors, 18] electrode in lithium ion batteries, [16, 19] and magnetic storage media. 20 Also, studies on $\mathrm{CuO}$ have shown strong dependence of its properties on quantum size effects, 21, 22. and has recently been shown to exhibit multiferroicity at $\mathrm{T}_{c} \sim 230 \mathrm{~K} .23,24$,

Experimentally, $\mathrm{CuO}$ has a monoclinic crystal structure with $\mathrm{C} 2 / \mathrm{c}$ symmetry. [25, 26] It has eight (8) formula units per magnetic unit cell. It is further reported to be a $p$-type semiconductor with band gap of 1.0 $1.9 \mathrm{eV}$ [15, 27-29] and local moment per $\mathrm{Cu}$ atom of $\sim 0.7 \mu_{B}$. [10, 30] Standard density functional theory (DFT) with local exchange-correlation functionals (see for e.g., Refs 31 33) generally predict a nonmagnetic ground state with metallic character instead of the well-known semiconducting ground state. The failure of standard DFT to obtain the correct electronic properties of $\mathrm{CuO}$ should be understood from its intrinsic nature (inability to treat electron-electron interactions in the so-called correlated systems). Improvements in first-principle theories based on density functional theory (DFT) plus screened Coulomb interaction (U) (DFT $+\mathrm{U})$ as proposed, developed, and utilized by Anisimov and co-workers 12, 13, 34, 35 has remedied this situation. While this method has been used to study the band structure of $\mathrm{CuO}$ (see for e.g., Refs. [12, 13, 36]), we are not aware of any optical study of $\mathrm{CuO}$ utilizing this method. 
There have been many theoretical[12, 24, 36, 41] and experimental studies [10, 23, 25, 27, 42,44 of $\mathrm{CuO}$. The purpose of this paper is to present optical properties of $\mathrm{CuO}$ based on the modern electronic structure that yield electronic properties of the strongly correlated systems in agreement with experiments, 12, 13, 36, in contrast to standard density functionals. Therefore, enables direct, quantitative comparisons of band structures and optical properties with experiment, without any adjustments, such as scaling the magnitude of the absorption or applying scissors operators to fix the gap. We extensively discuss the results in relation to experimental data. We hope that they will motivate future experimental investigations of the band structure of $\mathrm{CuO}$ particularly using optical measurements and photoemission.

The rest of this article is organized as follows. After this introduction in section [1. the computational methods and details are given in section III. The results of our self-consistent calculations are presented and discussed in section [II]. We will then conclude in section IV.

\section{METHOD AND COMPUTATIONAL DETAILS}

One difficulty in the computation of material properties is that the band gaps and related properties of most materials are generally underestimated by the standard density functional theory (DFT) approximations. To avoid this, we utilized the density functional theory (DFT) plus the effective Coulomb interaction (U) (DFT $+\mathrm{U})$ formalism using the general potential linearized augmented planewave (LAPW) method, 45 as implemented in the WIEN2k code. [46 Unlike other DFT $+\mathrm{U}$ computations for the electronic properties of $\mathrm{CuO}$ [24, 36, 41] that utilized the effective U-value $\left(\mathrm{U}_{\text {eff }}\right)$ calculated for e.g., $\mathrm{CaCuO}_{2} 13$ and $\mathrm{La}_{2} \mathrm{CuO}_{4}$, 47] in our case, we have self-consistently computed the $\mathrm{U}_{\text {eff }}$ unique to $\mathrm{CuO}$ using the constrained $\mathrm{DFT}+\mathrm{U}$ scheme of Anisimov et al. 48 as implemented by Madsen et al. 49] in WIEN2k code. [46] With this approach, the effective Coulomb interaction $\left(\mathrm{U}_{e f f}\right)$ on the $\mathrm{Cu} d$ state is calculated self-consistently. The computed value of $\mathrm{U}_{\text {eff }}$ is $7.14 \mathrm{eV}$. The DFT part of the computation utilized the Perdew-Burke-Ernzerhof generalized gradient approximation (PBE-GGA). 50.

Aside from the use of the DFT $+\mathrm{U}$ formalism, the other details of our calculations are standard. We used well converged basis sets with dense Brillouin zone samplings which is needed for the optical properties. For this purpose we used a uniform $48 \times 48 \times 48$ grid. The LAPW sphere radii are 1.92 and 1.71 bohr for $\mathrm{Cu}$ and $\mathrm{O}$. We utilized the room temperature experimental monoclinic crystal strucuture with lattice parameter, $\mathrm{a}=4.6837 \AA, \mathrm{b}$ $=3.4226 \AA$, and $\mathrm{c}=5.1288 \AA$. [26, 29] All calculations are performed relativistically. We carried out several sets of self-consistent calculations using different magnetic configurations. All attempts to obtain a ferromagnetic or

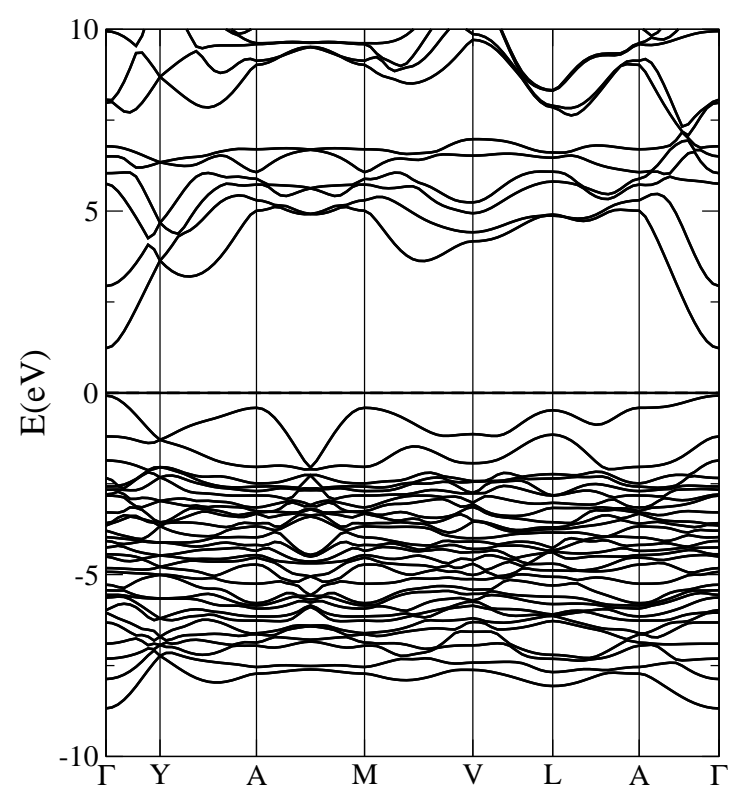

FIG. 1: (Color online) The calculated band structure of AFM $\mathrm{CuO}$. The horizontal straight line is the position of the Fermi energy $\left(E_{F}\right)$ which has been set equal to zero.

non-magnetic solutions always led to an antiferromagnetic ground state. 6, 8, 12, 13, This is in agreement with other results that show that $\mathrm{CuO}$ is an antiferromagnetic insulator. Since spin-orbit coupling (SOC) is important for $\mathrm{CuO}$, reported results are for DFT $+\mathrm{U}+$ SOC. As mentioned, we apply no scissors operators or other adjustments.

\section{RESULTS AND DISCUSSION}

\section{A. Band Structure}

We begin with the calculated band structure in relation to experimental data. Our calculated band structure for $\mathrm{CuO}$ is given in Fig. 1. The band structure is qualitatively similar to those reported previously, but there are quantitative differences resulting from the use of the $\mathrm{DFT}+\mathrm{U}$ functional, and these are important.

First of all, we note the values of the band gap. We find band gap $E_{g}=1.25 \mathrm{eV}(1.251 \mathrm{eV})$. The calculated band gap is in accord with experimentally reported ambient temperature band gap value in the range 1.0 to $1.9 \mathrm{eV}$. [15, 27 29] The hybrid functional results of Rocquefelte et al.37 reported a band gap of $1.42 \mathrm{eV}$ (for mixing ratio $\alpha=0.15$ ) and $2.4 \mathrm{eV}$ (for mixing ratio $\alpha>$ 0.15). The Green function plus screened Coulomb interation $(\mathrm{GW})$ and $\mathrm{GW}$ plus on-site potential $\left(\mathrm{GW}+\mathrm{V}_{d}\right)$ work of Lany 51 reported values of 2.42 and $1.40 \mathrm{eV}$, respectively. 
We did calculations as a function of the screen Coulomb values around the self-consistently determined value of $7.14 \mathrm{eV}\left(U_{\text {eff }}=5,6,7,8 \mathrm{eV}\right)$. Utilizing these $U_{\text {eff }}$ values to carry-out self-consistent computations, we only noticed a change of $0.12 \mathrm{eV}$ in the band gap, we conclude that this is too little to cause any significant change in the band gap of $\mathrm{CuO}$. We also carefully checked the dependence of the gap on various computational parameters, such as energy cut-offs, energy window size for the SOC calculation, LAPW sphere radii and Brillouin zone sampling. We find that at worst, the errors related to these are less than $0.08 \mathrm{eV}$ in the gap. In both cases, the position of the valence band maximum and the conduction band minimum didn't change.

The calculated magnetic moment per $\mathrm{Cu}$ atom $\left(\mathrm{M}_{C u}\right)$ (in the units of $\mu_{B}$ ) is $0.68 \mu_{B}$ in good agreement with the experimental reported value of $\sim 0.7 \mu_{B}$. 10, 30 We note that the magnetic moment per oxygen atom $\left(\mathrm{M}_{O}\right)$ is significant. It is $0.18 \mu_{B}$ in good agreement with experimental value of $0.14 \mu_{B}$. 11] We note that the hybrid functional results of Rocquefelte et al. 37 reported $\mathrm{M}_{C u}$ and $\mathrm{M}_{O}$ value of 0.65 and $0.12 \mu_{B}$ (for mixing parameter $\alpha=0.15$ ), while $\mathrm{M}_{C u}=0.74$ and $\mathrm{M}_{O}=0.09 \mu_{B}$ (for $\alpha>$ $0.15)$, respectively.

\section{B. Comparison with Photoemission Spectra}

Photoemission spectra (PES) experiments provide a direct measure of the electronic structure of the occupied states. There have been PES experiments for $\mathrm{CuO}$, with which we can compare with. 1, 44, 52, 54

In Fig. 2, we show the computed density of states (DOS) (Fig 2(a)) and the partial density of states (Figs. 2(b) to 2(c)) of $\mathrm{CuO}$ for both the spin majority and minority channels. While the bands are derived from a strong hybridization between $\mathrm{Cu} 3 d$ and $\mathrm{O} 2 p$ states, the conduction band (up to $\sim 3.56 \mathrm{eV}$ ) in the proximity of the Fermi level are formed mainly by the $\mathrm{Cu} 3 d(\mathrm{Cu}$ $3 d_{x^{2}-y^{2}} \downarrow$ ) states (c.f. Fig 2(b)). This corresponds to a case where there is a hole in the $\mathrm{Cu} 3 d_{x^{2}-y^{2}}$ states, the so-called $3 \mathrm{~d}^{9}$ configuration (we have used the local coordinate system centered on $\mathrm{Cu}$ where $\mathrm{x}$ and $\mathrm{y}$ axes are directed approximately to four neighboring oxygen). In one of the earliest ab-initio study of orbital decomposition in $\mathrm{CuO}$, 13 it was noted that the minority spin $(\mathrm{Cu}$ $\left.3 d_{x^{2}-y^{2}} \downarrow\right)$ states contribute strongly to the conduction band minimum and the (majority spins) $\mathrm{Cu} 3 d_{x^{2}-y^{2}} \uparrow$ and the $\mathrm{O} 2 p$ states constitute the bands in the valence band maximum. While the bands in the proximity of the Fermi energy $\left(\mathrm{E}_{F}\right)$ are predominantly $\mathrm{Cu} 3 d_{x^{2}-y^{2}} \downarrow$, there is a significant contribution from the $\mathrm{O} 2 p$ states. As it is evident from Figs. 2(b) and (c), the spin polarization of the states around the Fermi energy arise entirely from $\mathrm{Cu} 3 d_{x^{2}-y^{2}}$ states. One can reconcile the various results that claim the non-detection of the $3 d^{9}$ configuration (see for e.g., Ref. 56 ) with those that actually see it, by noting that the $3 d^{9}$ configuration is very compli-

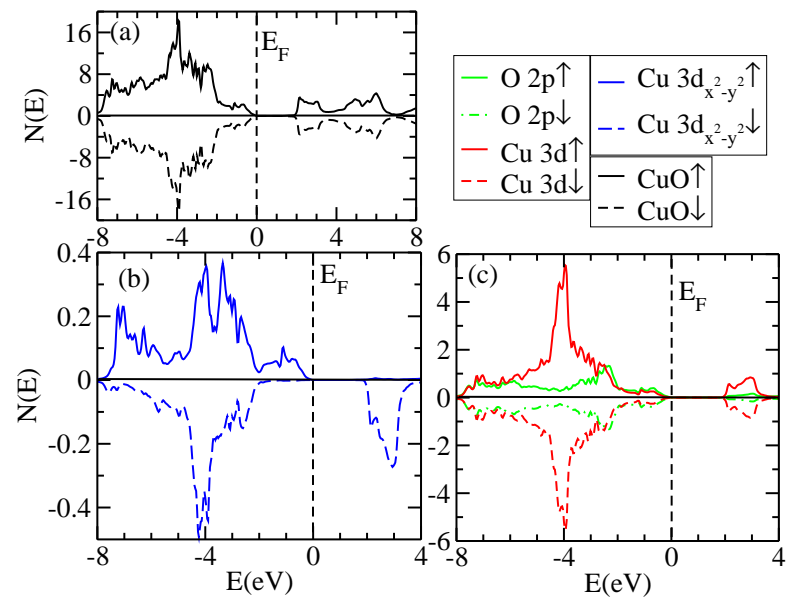

FIG. 2: (Color online) (a) The calculated total density of states of AFM CuO. (b) The calculated projected density of states of the $x^{2}-y^{2} \mathrm{Cu} 3 d$ states. (c) The calculated projected density of states of the $\mathrm{O} 2 p$ and $\mathrm{Cu} 3 d$ states. Figure 2(b) has been plotted using the local coordinate system centered on $\mathrm{Cu}$ where $\mathrm{x}$ and $\mathrm{y}$ axes are directed approximately to four neighboring oxygen. The vertical straight line is the position of the Fermi energy $\left(\mathrm{E}_{\mathrm{F}}\right)$ which has been set equal to zero.

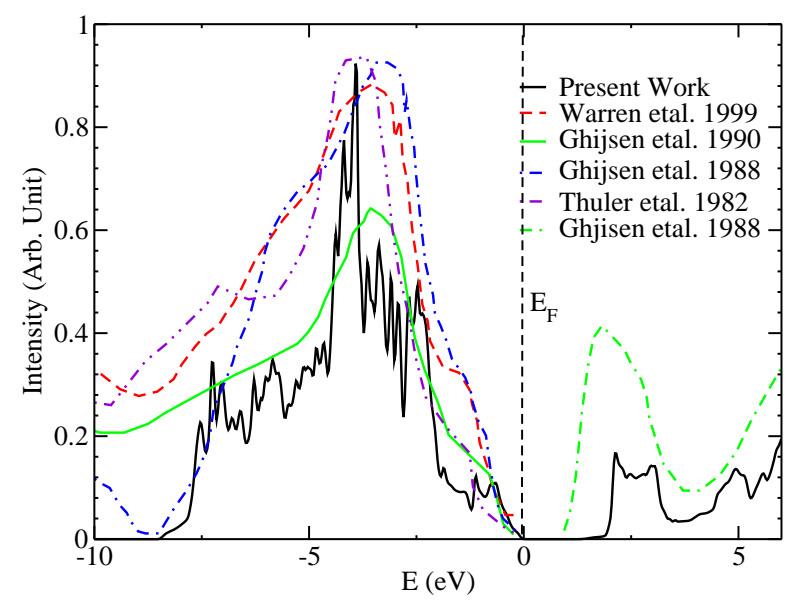

FIG. 3: (Color online) Calculated AFM CuO valence and low energy conduction band spectra without broadening as compared with experimental spectra. 1, 44, 53, 55. The experimental data are: the PES study of Warren et al. 44] (plot key Warren etal. 1999), the resonant PES study of Ghijsen et al. 55 (plot key Ghijsen etal. 1990), the PES study of Ghijsen et al. 1] (measured with He II radiation) (plot key Ghijsen etal. 1998), and the PES study of Thuler et al. 53. (plot key Thuler etal. 1982). The vertical straight line is the position of the Fermi energy which has been set equal to zero. 
cated [11, 56, 57] and observing it both in experiments and computations require very careful handling of the orbital decomposition. The most prominent feature in the conduction band are broad peaks at 2.13, 3.02, 4.97, and $5.98 \mathrm{eV}$. There are shallow minima around $3.62 \pm 0.33$ and $5.29 \mathrm{eV}$.

The states around the valence band maximum are predominantly of the $\mathrm{O} 2 p$ states hybridizing with the $\mathrm{Cd}$ $d$ (only the $\mathrm{Cu} 3 d_{x^{2}-y^{2}} \uparrow$ ) states in agreement with PES result of Warren et al. 44, resonant and X-ray photoemission spectroscopy (XPS) results of Shen et al. $[52$, and the X-ray photoemission spectroscopy results of Ghijsen et al. [1. One can thus conclude that the states in the proximity of the Fermi energy are basically huge complex band of entirely the $\mathrm{Cu} 3 d_{x^{2}-y^{2}}$ and $\mathrm{O} 2 p$ states. The most prominent structure in the valence states is a peak at $\sim 3.93 \mathrm{eV}$. This peak is exclusively derived from the $\mathrm{Cu} 3 d$ states.

Figure 3 shows the comparison of our computed DOS with experiments. We reiterate that our spectra were not shifted to coincide with experiment contrary to what is often done in such comparisons with experiments (e.g. Ref. 31). As can be seen from Fig. 3, the overall valence band spectra agree well with experimental ones. In particular, the agreement between our computed results and experimental spectra is reasonably good, both in terms of the relative intensities of the resonances and their binding energy positions. We note that there may be more features in our computed spectra as no broadening is used. Small deviation from experiment can be attributed to the fact that the intensity of photoemission spectra (PES) depend very sensitively on the photoionization across sections of the atomic sublevels. This deviation can even be seen between different experimental results.

The features from 0 to $\approx 8.0 \mathrm{eV}$ below $\mathrm{E}_{F}$ are predominantly due to $\mathrm{Cu} 3 d$ and $\mathrm{O} 2 p$ states in basic agreement with the x-ray photoelectron spectroscopy (XPS) and reflection electron energy-loss spectroscopy (REELS) results of Tahir et al. 27. We calculate the valence band width to be $\approx 8.41 \mathrm{eV}$ and the position of the maximum of the valence band is locate at $\approx 3.83 \mathrm{eV}$ in basic agreement with experimental ones with reported band width of 7.8 $-8.5 \mathrm{eV}$ and the position of the valence band maximum located at $3-4 \mathrm{eV}$. [1, 44, 52, 53, 55, Taking a closer look at the valence bands in the proximity of the Fermi energy, we find that these states are highly localized. We predict peaks at $\sim 0.66 \mathrm{eV}$ and $1.10 \mathrm{eV}$. This feature is reminiscent of the so-called ${ }^{1} A_{1 g}$ singlet. 44, 52, 55, 58, This singlet is formed due to the hybridization of $\mathrm{Cu} 3 d$ (mainly the $\mathrm{Cu} 3 \mathrm{~d}_{x^{2}-y^{2}}$ ) and $\mathrm{O} 2 p$ states. [59] It is the so-called $\mathrm{d}^{9} \underline{\mathrm{L}}^{1}$ (where $\underline{L}$ is the ligand oxygen hole) final state with one hole in the $3 \mathrm{~d}_{x^{2}-y^{2}}$ orbital and the other in an $\mathrm{O} 2 p$ orbital. 11, 58. We predict antiresonance dip around $1.23 \pm 0.12 \mathrm{eV}$ in basic agreement with experiment 44 and a peak at $2.44 \pm 0.21 \mathrm{eV}$. There are cluster of shoulders at $3.09 \pm 0.22$ and $5.2 \pm 0.32 \mathrm{eV}$. The ultraviolet photoemission data of Ghijsenet al. [1, 55] reported
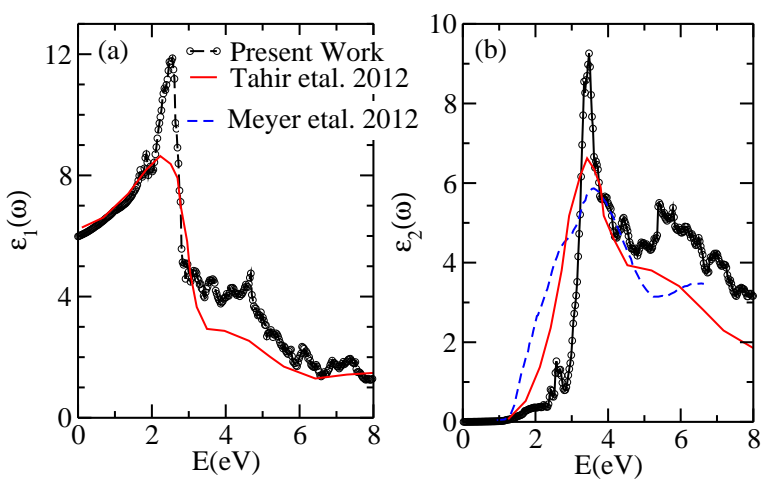

FIG. 4: (Color online) (a) Calculated, dispersive part, $\varepsilon_{1}(\omega)$, of the dielectric function of AFM CuO. (b) Calculated, absorptive part, $\varepsilon_{2}(\omega)$, of the dielectric function of $\mathrm{CuO}$. Both $\varepsilon_{1}(\omega)$ and $\varepsilon_{2}(\omega)$ are compared to the experimental spectra of Tahir et al. 27. Note, both spectra have not been broadened as such, may have more structure than the experimental ones.

features at $1.23 \pm 0.11,3.10 \pm 0.11$, and $5.50 \pm 0.40 \mathrm{eV}$.

The low energy conduction band spectra show good agreement with the inverse photoemission (Bremsstrahlung isochromat spectroscopy) spectra experiment of Ghijsen et al. [1]. Our computation predicted peaks at $2.11,2.98 \pm 0.20$, and $4.97 \mathrm{eV}$.

\section{Optical Properties and Comparison with Experiment}

Optical spectroscopy, while less direct than PES, provides detailed information about the electronic structure and has the advantage of being a true bulk probe.

The optical properties of $\mathrm{CuO}$ in both film and bulk have been studied using several experimental methods. [27, 28, 60, 63. Most of the studies in the visible and ultraviolet have been near-normal incidence reflectivity measurements. 28, 60, 62] These methods have certain potential sources of errors. In general, they involve multiple measurements to obtain the dielectric constants and/or Kramers-Kronig (KK) analysis, which can suffer from uncertainty in the absolute amplitude. This is associated with extrapolations necessary for the KK transformation of experimental reflectivity spectra.

Spectroscopic ellipsometry (SE) is one of the parallel measurement techniques that avoids these problems in measuring the optical constants of solids. 64 The big advantage of the SE technique, and other parallel measurement techniques, is that both the real and imaginary part of the complex dielectric function of a material may be obtained directly as a function of wavelength without requiring multiple measurements or KK analysis. One of the earliest $\mathrm{SE}$ measurements for $\mathrm{CuO}$ are those of 
Ito and co-workers 65 in the $1.2-5.0 \mathrm{eV}$ photon-energy range at room temperature, Meyer and co-workers, 66. and Nomerovannaya and co-workers. 67.

As previously noted, we calculated optical properties based on our DFT + U electronic structure, with no adjustment. This was done using the optical properties package of the WIEN2k code. While it is conventional to plot calculated optical data with a broadening added to mimic experimental data, we instead show results with no added broadening in order to show more clearly the features in the calculated spectra. Our calculated dispersive part of the dielectric function, $\varepsilon_{1}(\omega)$ and the absorptive part, $\varepsilon_{2}(\omega)$ are shown in Fig. 4 in comparison with the experimental results of Tahir and Tougaard [27] and Meyer et al. 66. As can be seen, our computed spectra for $\varepsilon_{1}(\omega)$ (c.f. Fig. $\left.4(\mathrm{a})\right)$ and $\varepsilon_{2}(\omega)$ (c.f. Fig. $4(\mathrm{~b})$ ), are in good agreement with experiment.

There is no yet rigorous ab-initio optical property study of $\mathrm{CuO}$ especially, taking into account the strong electron-electron correlations; as such, this study serves as a baseline for comparing with future experiment and theory.

Figure 4(a) shows our calculated dispersive part of the dielectric function, $\varepsilon_{1}(\omega)$ in comparison with the data of Tahir et al. [27] and Meyer et al. [66. The main experimental features in $\mathrm{CuO}$ [27] are reproduced in our results. The main feature is a shoulder at $1.82 \pm 0.2 \mathrm{eV}$, followed by a steep rise. We predict cluster of small shoulders at $3.05 \pm 0.42 \mathrm{eV}$. Our data show a dip at $\sim 3.82 \mathrm{eV}$. Experiment show a similar dip at $\sim 3.68 \mathrm{eV}$. 27] The SE data of Ito et al. 65] show features at $\sim 1.6,2.0,2.6$, and $3.4 \mathrm{eV}$. 65. We predict a shoulder at $\sim 6.62 \pm 0.21$ $\mathrm{eV}$ followed by a steep decrease slowly towards almost zero at higher energies. Our computed $\varepsilon(\infty)=\Re(\varepsilon(0))$ is 6.12. The infrared spectroscopy data of Kuz'menko et al. 68] reported $\varepsilon(\infty)$ of $5.9-7.8$ (average value is 6.60 ), the SE data of Ito et al. 65] reported a value of 6.45, and the polarized reflectance of a single crystal of $\mathrm{CuO}$ study of Homes et al. 69] reported a value of $6.2-6.3$. We note that the $\mathrm{GW}+\mathrm{V}_{d}$ results of Lany [51] reported static electronic dielectric constant of 7.9. The maximum amplitude of $\varepsilon_{1}(\omega)$ is $11.79 \pm 0.15$ at $2.52 \pm 0.13 \mathrm{eV}$.

Figure 4(b) shows our calculated absorptive part of the dielectric function, $\varepsilon_{2}(\omega)$ in comparison with the experi- ments. 27]. We found a very small kink around $1.51 \mathrm{eV}$ and then, shoulders around 2.40 and $2.61 \mathrm{eV}$, followed by other sets of close dips around 2.30, 2.53, and 2.82 $\mathrm{eV}$; after which, the spectra increased with a steep rise in energy. The maximum of $\varepsilon_{2}(\omega)$ is $\sim 8.96$ at energy of $3.57 \mathrm{eV}$. The experimental data of Meyer et al. 66] show features at 1.66, 2.07, $2.68 \mathrm{eV}$ with a maximum at 3.46 $\mathrm{eV}$ in good agreement with our computed results. We also find shoulders at $3.86,4.45$, and $5.41 \mathrm{eV}$; and cluster of dips at 4.24 , and $4.85 \pm 0.32 \mathrm{eV}$.

Thus there is a very close correspondence between our first principles results, which represent standard band structure descriptions, and both optical data from SE and PES measurements.

\section{CONCLUSION}

We have performed self-consistent DFT $+\mathrm{U}$ to study the electronic and optical properties of monoclinic $\mathrm{CuO}$. Our computations show that there is significant $\mathrm{Cu}-d$ and O- $p$ hybridization of the states in the proximity of the Fermi energy. Our calculated electronic structure and optical spectra are in good agreement with experiments. We obtain a band gap of $1.25 \mathrm{eV}$ and local moment per $\mathrm{Cu}$ atom is $0.68 \mu_{B}$ with significant magnetic moment of $\mathrm{O} \sim 0.18 \mu_{B}$. The comparison of our calculated electronic structure with PES show generally good agreement with the key binding energies correctly obtained in our computation. So is our computed optical spectra.

Acknowledgments. Work at LSU is funded in part by the National Science Foundation award No. EPS1003897. V. I. Anisimov acknowledgments the supports of the following. The Russian Foundation for Basic Research (Projects No. 13-02-00050 and No. 12-02-91371$\mathrm{CT}_{a}$ ), the fund of the President of the Russian Federation for the support of scientific schools NSH-6172.2012.2, the Program of the Russian Academy of Science Presidium "Quantum microphysics of condensed matter" 12-P2-1017, and the grant of the Ministry of education and science of Russia No. 14.A18.21.0076. High performance computational resources were provided by Louisiana Optical Network Initiative (LONI). C. E. Ekuma wishes to thank the Government of Ebonyi State, Nigeria.
[1] J. Ghijsen, L. H. Tjeng, J. van Elp, H. Eskes, J. Westerink, G. A. Sawatzky, and M. T. Czyzyk, Phys. Rev. B 38, 11322-11330 (1988).

[2] F. Parmigiani and G. Samoggia, Europys. Lett. 7(6), 543 (1988).

[3] X. G. Zheng, C. N. Xu, Y. Tomokiyo, E. Tanaka, H. Yamada, and Y. Soejima, Phys. Rev. Lett. 85, 5170-5173 (2000).

[4] J. G. Bednorz and K. A. Müller, Rev. Mod. Phys. 60, 585-600 (1988).

[5] J. Bednorz and K. Müller, Z. Physik B Conden. Matter
64(2), 189-193 (1986).

[6] L. F. Mattheiss, Phys. Rev. B 5, 290-306 (1972).

[7] K. Terakura, T. Oguchi, A. R. Williams, and J. Kübler, Phys. Rev. B 30, 4734-4747 (1984).

[8] W. A. Harrison, Phys. Rev. B 76, 054417 (2007).

[9] T. Shimizu, T. Matsumoto, A. Goto, T. V. Chandrasekhar Rao, K. Yoshimura, and K. Kosuge, Phys. Rev. B 68, 224433 (2003).

[10] B. X. Yang, T. R. Thurston, J. M. Tranquada, and G. Shirane, Phys. Rev. B 39, 4343-4349 (1989).

[11] J. B. Forsyth, P. J. Brown, and B. M. Wanklyn, J. Phys. 
C: Solid State Physics 21(15), 2917 (1988).

[12] V. I. Anisimov, F. Aryasetiawan, and A. I. Lichtenstein, J. Phys.: Condens. Matter 9, $767-808$ (1997).

[13] V. I. Anisimov, J. Zaanen, and O. K. Andersen, Phys. Rev. B 44, 943-954 (1991).

[14] P. Poizot, S. Laruelle, S. Grugeon, and J. M. Taracon, Nature 407, 496 (2000).

[15] S. C. Ray, Sol. Energy Mater. Sol. Cells 68, 307 (2001).

[16] T. Ben-Moshe, I. Dror, and B. Berkowitz, Appl. Catal. B 85, 207-211 (2009).

[17] T. Ishihara, M. Higuchi, T. Takagi, M. Ito, H. Nishiguchi, and Y. Takita, J. Mater. Chem. 8, 2037-2042 (1998).

[18] Y. Jiang, S. Decker, C. Mohs, and K. J. Klabunde, J. Catal. 180, 24 (1998).

[19] J. Morales, L. Sánchez, F. Martín, R.-B. J. R, and M. Sánchez, Electrochim Acta 49, 4589-459 (2004).

[20] R. V. Kumar, Y. Diamant, and A. Gedanken, Chemistry of Materials 12(8), 2301-2305 (2000).

[21] K. Borgohain, J. B. Singh, M. V. Rama Rao, T. Shripathi, and S. Mahamuni, Phys. Rev. B 61, 11093-11096 (2000).

[22] S. Rehman, A. Mumtaz, and S. K. Hasanain, J. Nanopart. Res. 13, 2497-2507 (2011).

[23] T. Kimura, Y. Sekio, H. Nakamura, T. Siegrist, and A. P.Ramirez, Nature Mater. 7, 291 (2008).

[24] G. Jin, K. Cao, G.-C. Guo, and L. He, Phys. Rev. Lett. 108, 187205 (2012).

[25] A. A. Samokhvalov, N. N. Loshkareva, Y. P. Sukhorukov, V. A. Gruverman, B. A. Gizhveskii, and N. M. Chebotaev, JETP Lett. 49, 523 (1989).

[26] ICSD, Inorganic Crystal Structure Database (ICSD), National Institute of Standards and Technology (NIST) Release 2013/1, vol. 1 (NIST, 2013).

[27] D. Tahir and S. Tougaard, J. Phys.: Conden. Matter 24(17), 175002 (2012).

[28] F. Marabelli, G. B. Parravicini, and F. Salghetti-Drioli, Phys. Rev. B 52, 1433-1436 (1995).

[29] O. Madelung, U. Rössler, and M. Schulz (eds.) Numerical Data and Functional Relationships in Science and Technology, Landolt-Börnstein, New Series, Group III, vol. 17a, and 22a (Springer, 2006).

[30] M. Ain, A. Menelle, B. M. Wanklyn, and E. F. Bertaut, J. Phys.: Conden. Matter 4(23), 5327 (1992).

[31] W. Y. Ching, Y.-N. Xu, and K. W. Wong, Phys. Rev. B 40, 7684-7695 (1989).

[32] C. Kanagaraj and N. Baskaran, Advanced Materials Research 488-489, 129 (2012).

[33] M. Heinemann, B. Eifert, and C. Heiliger, Phys. Rev. B 87, 115111 (2013).

[34] M. T. Czyżyk and G. A. Sawatzky, Phys. Rev. B 49, 14211-14228 (1994).

[35] A. I. Liechtenstein, V. I. Anisimov, and J. Zaanen, Phys. Rev. B 52, R5467-R5470 (1995).

[36] D. Wu, Q. Zhang, and M. Tao, Phys. Rev. B 73, 235206 (2006).

[37] X. Rocquefelte, M.-H. Whangbo, A. Villesuzanne, S. Jobic, F. Tran, K. Schwarz, and P. Blaha, J. Phys.: Conden. Matter 22(4), 045502 (2010).

[38] W. Siemons, G. Koster, D. H. A. Blank, R. H. Hammond, T. H. Geballe, and M. R. Beasley, Phys. Rev. B 79, 195122 (2009).

[39] G. Giovannetti, S. Kumar, A. Stroppa, J. van den Brink, S. Picozzi, and J. Lorenzana, Phys. Rev. Lett. 106,
026401 (2011).

[40] P. Tolédano, N. Leo, D. D. Khalyavin, L. C. Chapon, T. Hoffmann, D. Meier, and M. Fiebig, Phys. Rev. Lett. 106, 257601 (2011).

[41] B. Himmetoglu, R. M. Wentzcovitch, and M. Cococcioni, Phys. Rev. B 84, 115108 (2011).

[42] B. A. Gizhevskii, A. S. M. Yu. P. Sukhorukov, N. N. Loshkareva, E. V. Mostovshchikova, A. E. Ermakov, E. A. Kozlov, M. A. Ǚmin, and V. S. Gaviko, J. Exp. and Theor.Phys. 102, 298 (2006).

[43] B. A. Gizhevskii, Y. P. Sukhorukov, N. N. Loshkareva, A. S. Moskvin, E. V. Zenkov, and E. A. Kozlov, J. Phys.: Conden. Matter 17(3), 499 (2005).

[44] S. Warren, W. R. Flavell, A. G. Thomas, J. Hollingworth, P. L. Wincott, A. F. Prime, S. Downes, and C. Chen, J. Phys.: Conden. Matter 11(26), 5021 (1999).

[45] D. J. Singh, Planewaves, Pseudopotentials, and the LAPW Method, 2nd Ed. (Springer-Velag, Berlin, 2006).

[46] P. Blaha, K. Schwarz, G. Madsen, D. Kvasnicka, and J. Luitz, WIEN2K, An Augmented Plane Wave+Local Orbitals Program for Calculating Crystal Structure (K. Schwarz Technical University, Wien, Austria, 2001).

[47] M. Cococcioni and S. de Gironcoli, Phys. Rev. B 71, 035105 (2005).

[48] V. I. Anisimov and O. Gunnarsson, Phys. Rev. B 43, 7570 (1991).

[49] G. K. H. Madsen and P. Novark, Europys. Lett. 69, 777 (2005).

[50] J. P. Perdew, K. Burke, and M. Ernzerhof, Phys. Rev. Lett. 77, 3865 (1996).

[51] S. Lany, Phys. Rev. B 87, 085112 (2013).

[52] Z. X. Shen, R. S. List, D. S. Dessau, F. Parmigiani, A. J. Arko, R. Bartlett, B. O. Wells, I. Lindau, and W. E. Spicer, Phys. Rev. B 42, 8081 (1990).

[53] M. R. Thuler, R. L. Benbow, and Z. Hurych, Phys. Rev. B 26, 669 (1982).

[54] L. H. Tjeng, B. Sinkovic, N. B. Brookes, J. B. Goedkoop, R. Hesper, E. Pellegrin, F. M. F. de Groot, S. Altieri, S. L. Hulbert, E. Shekel, and G. A. Sawatzky, Phys. Rev. Lett. 78, 1126-1129 (1997).

[55] J. Ghijsen, L. H. Tjeng, H. Eskes, G. A. Sawatzky, and R. L. Johnson, Phys. Rev. B 42, 2268-2274 (1990).

[56] M. A. Mäki-Jaskari, Modelling and Simulation in Materials Science and Engineering 14(2), 207 (2006).

[57] M. Takahashi and J.-i. Igarashi, Phys. Rev. B 56, 12818 12824 (1997).

[58] H. Eskes, L. H. Tjeng, and G. A. Sawatzky, Phys. Rev. B 41, 288-299 (1990).

[59] A. K. McMahan, R. M. Martin, and S. Satpathy, Phys. Rev. B 38, 6650-6666 (1988).

[60] H. Wieder and A. W. Czanderna, J. Appl. Phys. 37, 184 (1966).

[61] D. Jundale, P. Joshi, S. Sen, and V. Patil, J. Mater. Sci.: Materials in Electronics 23(8), 1492-1499 (2012).

[62] A. Chen, H. Long, X. Li, Y. Li, G. Yang, and P. Lu, Vacuum 83(6), 927 - 930 (2009).

[63] B. Balamurugan and B. Mehta, Thin Solid Films 396(12), 90 - 96 (2001).

[64] D. E. Aspnes, in Handbook of Optical Constants of Solids (Academic, New York, 1985).

[65] T. Ito, , H. Yamaguchi, T. Masumi, and S. Adachi, J. Phys. Soc. Jpn. 67, 3304-3309 (1998).

[66] B. K. Meyer, A. Polity, D. Reppin, M. Becker, P. Her- 
ing, P. J. Klar, T. Sander, C. Reindl, J. Benz, M. Eickhoff, C. Heiliger, M. Heinemann, J. Bläsing, A. Krost, S. Shokovets, C. Mller, and C. Ronning, physica status solidi (b) 249(8), 1487-1509 (2012).

[67] L. V. Nomerovannaya, A. A. Makhnev, M. M. Kirillova, S. V. Moshkin, M. G. Lyubimov, and N. V. Egorova, Superconductivity 3, 159 (1990).
[68] A. B. Kuz'menko, D. van der Marel, P. J. M. van Bentum, E. A. Tishchenko, C. Presura, and A. A. Bush, Phys. Rev. B 63, 094303 (2001).

[69] C. C. Homes, M. Ziaei, B. P. Clayman, J. C. Irwin, and J. P. Franck, Phys. Rev. B 51, 3140-3150 (1995). 